\title{
Complicaciones periodontales en las leucemias en edad pediátrica
}

\author{
QUASSO L* \\ SCIPIONI C** \\ PAVESI L* \\ CALZAVARA IMANTOVANI D* \\ PEREA GARCÍA IM A \\ BASCONES MARTÍNEZ A
}

Quasso L, Scipioni C, Pavesi L, Calzavara Mantovani D, Perea García MA, Bascones Martínez A. Complicaciones periodontales en las leucemias en edad pediátrica. Av Periodon Implantol. 2005; 17, 2: 55-68.

\begin{abstract}
RESUMEN
El objetivo de este trabajo es analizar las complicaciones odonto-periodontales que se manifiestan en los pacientes pediátricos afectados por leucemias. En él se valora positivamente la intervención del odontólogo durante las fases iniciales de las leucemias, por las manifestaciones periodontales y neurológicas que se asocian a ellas, así como por las complicaciones periodontales y alteraciones odonto-esqueléticas originadas por las terapias antiblásticas.
\end{abstract}

Asimismo se hace una valoración de los tratamientos odontológicos para estos pacientes y a su educación en el ámbito higienico-preventivo oral que beneficia el pronóstico de estos pacientes.

Por último los autores enseñan los percentiles patológicos registrados en el período de observación (18 meses).

\section{PALABRAS CLAVE}

Leucemias, manifestaciones dento-periodontales de la cavidad oral, sección de hemato-oncología odontológica, prevención e higiene oral.

Aceptado para publicación: Mayo 2004

\section{INTRODUCCIÓN}

El odontólogo ha de figurar en todas las valoraciones de diagnóstico y tratamiento de la Leucemia en las diferentes fases de la enfermedad, ya que la Leucemia representa un tercio de los casos tumorales en edad pediátrica con una incidencia de 40 nuevos casos cada millón de habitantes y año (15-16\% de la población pediátrica total).
Por tanto, el Odontólogo toma un papel fundamental en todas las fases de la enfermedad, empezando por el diagnóstico interceptivo, durante las primeras manifestaciones orales de la enfermedad, así como apoyo en la rehabilitación del aparato estomatognático y durante las quimioterapias y las radioterapias antileucémicas. Además, para los pacientes sometidos a TMO (trasplante de médula ósea), el odontólogo se convierte en profesional de referencia en el diag- 
nóstico de complicaciones orales, como por ejemplo la GVHD (Graft Versus Host Disease) (1).

\section{PAPEL INTERCEPTIVO DEL ODONTÓLOGO EN LAS LEUCEMIAS: MANIFESTACIONES NEUROLÓGICAS DEL APARATO ESTOIMATOGNÁTICO}

Los pacientes afectados por Leucemia presentan, en el $69 \%$ de los casos, complicaciones en la cavidad oral; estas manifestaciones se pueden considerar como patognomónicas de la enfermedad en curso.

Tan sólo el 38\% de los pacientes refiere una sintomatologia subjetiva (2).

Por tanto, el papel interceptivo del odontólogo en las fases iniciales, donde se manifiesta la enfermedad, es de gran importancia.

Como sintomatología oral precoz de la enfermedad puede manifestarse un síndrome característico (aunque no patognomónico) llamado NUMB CHIN SYNDROME (NCS) o bien "Síndrome de la Formicación al mentón", causado por una lesión infiltrativa del área mandibular $(1,3,4)$.

La sintomatología de la NCS se puede resumir en:

- Parestesia o hipoestesia de la zona inervada por el n. mentoniano.

- Dolor en la zona del foramen mentoniano.

- Los dientes homolaterales a la lesión pueden presentar parestesia y dolor a la oclusión, a la percusión y/o a la movilidad, así como variaciones en la sensibilidad dental a las pruebas térmicas (menor o anulada).

- Posible extrusión bilateral de los molares puesta en evidencia con una mordida abierta.

- Radiográficamente, se presenta desaparición de los canales mandibulares bilateralmente, destrucción de la cresta alveolar, dilatación del espacio periodontal y disminución o pérdida de lámina dura $(1,4,5)$.

La NCS tal vez esté causada por:

- Compresión del nervio por infiltrados tumorales mandibulares.

- Invasión de las células tumorales en los nervios del cráneo.

- Implicación de la raíz trigeminal en los tumores metastásicos de las meninges $(1,4,5)$.

En ausencia de otras enfermedades odontológicas como quistes, flogosis o traumas de los huesos maxilares, se deberá pensar en la posibilidad de la existencia de un proceso leucémico e incluso en los casos de la desaparición de estas patologías o por remisión de la Leucemia, debido a la terapia (Figs. 1, 2, 3, 4, 5).

Otras manifestaciones dento-periodontales que aparecen con las leucemias se resumen en la Tabla $1(1,3)$ :

A consecuencia de la hipertrofia gingival, del sangrado y de la fragilidad del tejido gingival, muy raramente se puede manifestar Gingivitis ulcero-necrotizante (GUN). Esta representa, en las fases iniciales de las leucemias, una complicación grave.

Los factores que predisponen a la GUN puede ser una higiene oral inadecuada o una gingivitis persistente,

\section{TABLA 1.- MANIFESTACIONES ORALES DURANTE PATOLOGÍAS LEUCÉMICAS}

\section{SÍGNOS CLÍNICOS}

Hipertrofia gingival (foto 5)

Dolor al músculo esquelético

Parálisis del hipogloso.

Neuropatía del trigémino.

Tumefacciones de las glándulas salivales.

Numb Chin Syndrome

Mordida abierta anterior.

Parestesias.

Dolor al ocluir y al percutir los dientes

Petequias (Foto 1-4)

Hemorragia gingival

Fragilidad del tejido gingival

\section{SEÑALES RADIOGRÁFICAS}

Destrucción de la cresta alveolar.

Ampliación del espacio periodontal.

Desaparición del canal mandibular.

Fracturas patológicas.

Apiñamiento de los dientes.

Desaparición de la lámina dura.

Lesiones líticas y escleróticas del hueso. 


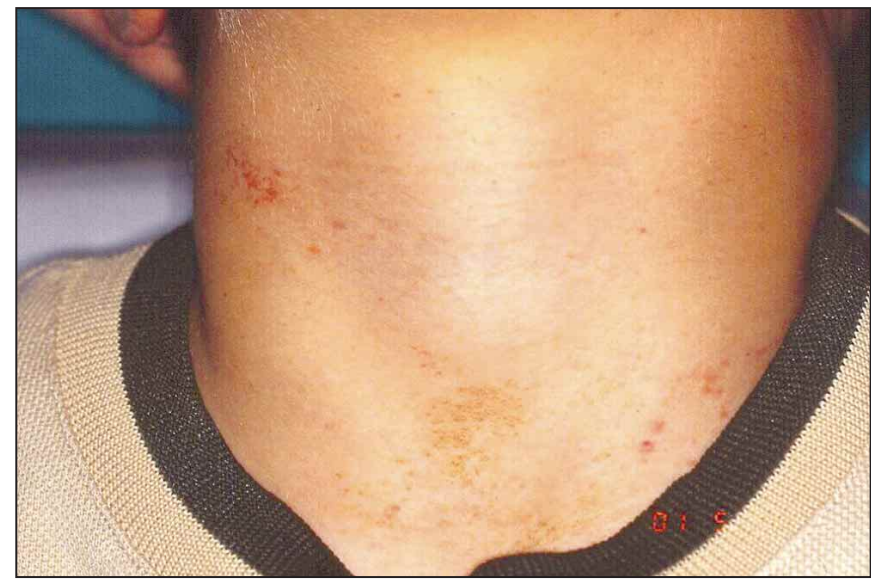

Fig. 1. Petequias hemorrágicas cutáneas.

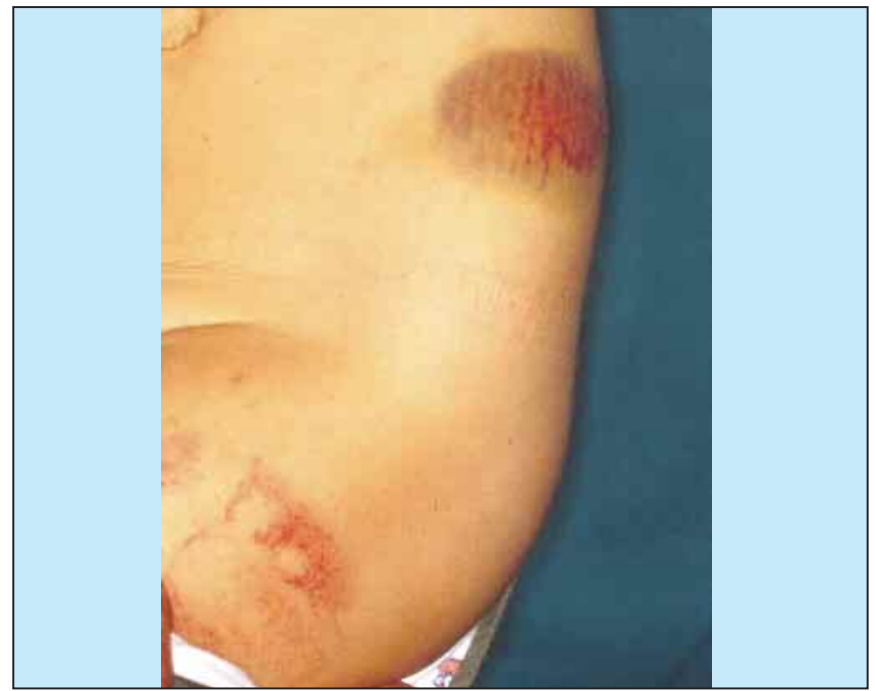

Fig. 2. Hematomas cutáneos en paciente trombocitopénico.

debido a que los pacientes leucémicos tienen dificultad en tener una buena higiene oral; sin embargo, la condición predisponente fundamental está representada precisamente por enfermedades sistémicas tal como la Leucemia así como por HIV, debido a una reducción de las defensas inmunitarias (6).

La GUN es una enfermedad dolorosa y repentina que comienza por las papilas interdentales y se difunde posteriormente a la encía produciendo necrosis y ulceraciones. Una señal clínica característica es la presencia de un eritema lineal que delimita las zonas ulceradas con respecto de los tejidos sanos.

Las úlceras están cubiertas por saburras blancas o amarillas formadas por leucocitos, eritrocitos, fibrina, tejido necrótico y bacterias.

El paciente además del dolor puede manifestar un mayor flujo salivar y foetor ex ore (7).

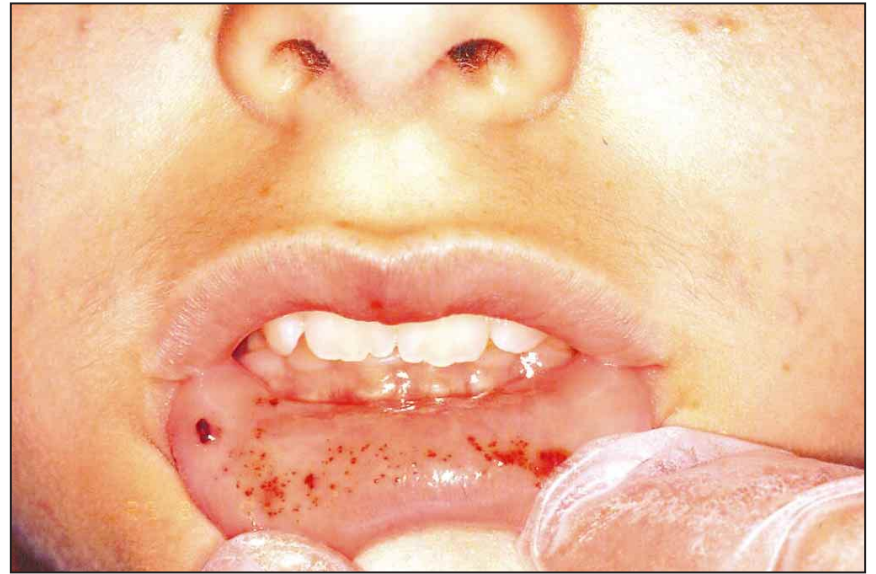

Fig. 3. Petequias hemorrágicas muco-labiales.

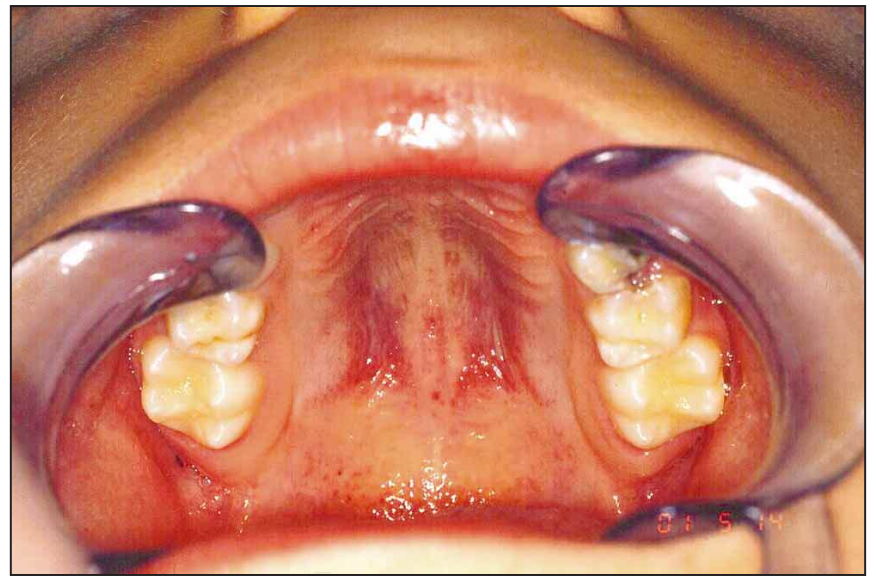

Fig. 4. Sufusiones hemorrágicas de la mucosa palatina.

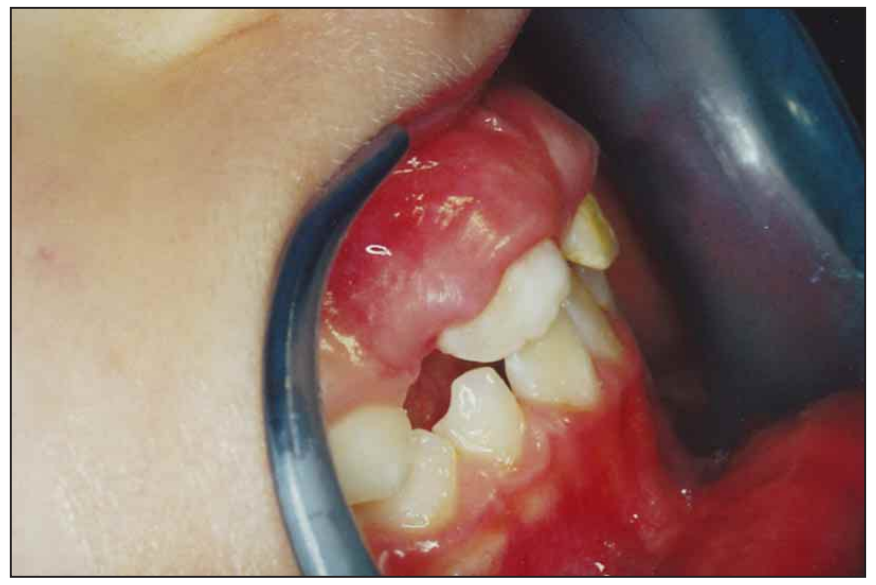

Fig. 5. Hipertrofia de la mucosa gingival.

Para el tratamiento de la GUN se aconseja, junto con una correcta práctica de higiene oral, enjuagues con clorhexidina y antisépticos, administración de metronidazol durante 7 días $(6,8)$.

Con referecia a la GUN, hemos de efectuar diagnostico 
diferencial con la Gingivostomatitis herpética, más frecuente en los niños. En este caso, la remoción de la placa y de la flora (formada por Espiroqueta fusiforme) disminuyen la inflamación y el dolor (9).

\section{IMANIFESTACIONES PERIODONTALES DURANTE LA QUIMIOTERAPIA Y LA RADIOTERAPIA}

Una vez diagnosticada la Leucemia, debe instaurarse terapia antiblástica.

Los protocolos terapéuticos varían según el tipo de leucemia a tratar. Siendo distintas según sea linfoide o mieloide y de la necesidad o no de intervenir con transplante de médula ósea (TMO).

Susbtancialmente las terapias antiblásticas pueden, en la mayoría de los casos, producir complicaciones más o menos importantes para la salud oral. En algunos casos las complicaciones pueden ser hasta graves y necesitar intervenciones urgentes tanto por problemas septicémicos o hemorrágicos.

Otras veces las reacciones de la mucosa oral pueden producir un dolor muy intenso, tanto como para necesitar la administración de morfina.

Los pacientes sometidos a ciclos terapéuticos de quimioterapia desarrollan alteraciones en la mucosa tan graves que se corre el peligro de obtener situaciones septicémicas graves, difíciles de controlar a nivel farmacológico, sobre todo en pacientes no sometidos preventivamente a limpieza de la cavidad oral. La mucosa oral puede alterarse por la patología presente (véase Tabla 1) así como los tratamientos indicados para erradicarlas

Los pacientes hemato-oncológicos pediátricos al estar afectados por esta enfermedad en edad escolar o preescolar, cuando la dentición aún no es definitiva con elementos dentales caducos, tal vez móviles, crea desgraciadamente la condición ideal para la formación de enfermedades de la cavidad oral, como: gingivitis, periodontitis, abscesos, flemones, hemorragias, sialorrea, etc. , determinando una menor higiene oral, ya que cada cepillado causa un dolor intenso y a menudo un abundante sangrado.

Situaciones del género susodicho pueden resultar muy peligrosas en pacientes jóvenes tratados con fármacos para las leucemias, donde la reducción de las defensas inmunitarias favorece a menudo la llegada de infecciones no solamente localizadas, sino también muy a menudo septicémicas (como ilustrado en el Gráfico 1).

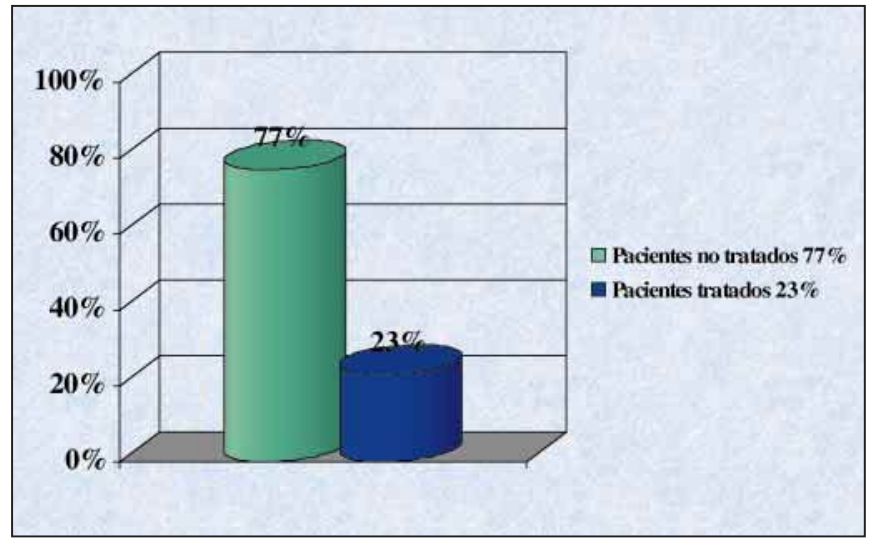

Gráfico 1: $A$ - Incidencia de septicemia en pacientes sometidos a terapia antiblástica sin tratamiento preventivo odontológico. B Incidencia de septicemia en pacientes sometidos a terapia antiblástica con tratamiento preventivo odontológico.

El riesgo de septicemias es muy elevado y según unos autores parece tener un origen oral en más del 50\% de los casos documentados $(24,25)$.

Otro problema subyacente que podría ocurrir es la ausencia de los síntomas inflamatorios, debido a la neutropenia, haciendo que el diagnóstico precoz de la infección sea más complicado y en consecuencia con un cierto índice de mortalidad $(13,17)$.

En estas situaciones patológicas tan variables, las bacterias saprófitas de la cavidad oral pueden adquirir agresividad debido a la disminución de los granulocitos y a las alteraciones de la mucosa oral en un ámbito global de mayor permeabilidad bacteriana causada por la terapia antiblástica $(12,13,16,17,18,19,20)$.

El papel del odontólogo es entonces muy importante, como profesional en colaboración con los terapeutas hemato-oncológicos, durante todas las fases de la enfermedad: diagnóstico, terapia, en el TMO y por fin en el seguimiento a largo plazo de los sobrevivientes.

\section{INCIDENCIA DE MANIFESTACIONES SEPTICÉMICAS}

Las curas odontológicas, en esta fase van orientadas al control de la higiene oral mediante: limpieza profesional, terapias conservadoras endodónticas indispensables, tartrectomía y alisado, corrección de restauraciones incongruentes, extracciones de elementos dentales móviles y de otros que podrán posteriormente ser substituidos. Por último, prevención odon- 


\section{TABLA 2.- MEDIDAS PREVENTIVAS DURANTE LA QUIMIOTERAPIA Y LA RADIOTERAPIA}

- Control mecánico de la placa (con cepillo y seda dental hasta que el recuento hemático sea adecuado).

- Control mecánico de la placa (limpieza con gasas y esponjas, enjuagues cuando el recuento hemático sea bajo).

- Control químico del ambiente oral (pasta de bicarbonato sódico y enjuagues con soluciones de bicarbonato y agua).

- Lubricación de los labios y de las mucosas (petrolato blanco, manteca de cacao).

- Prevención de la caries causada por la radioterapia (uso tópico de gel al flúor y a la clorhexidina).

- Profilaxis antiviral (Aciclovir).

- Profilaxis antifúngica (fluconazol y clorhexidina).

tológica clásicos como: selladores de los surcos dentales e ionoforesis con geles fluorados.

No se aconsejan terapias quirúgicas periodontales debido a la lenta curación de los tejidos.

Estas maniobras terapéuticas se activan hasta que los recuentos hemáticos no desciendan por debajo de los niveles de riesgo (leucocitos $<2.000 / \mathrm{mm}^{3}$ y trombocitos $<30.000 / \mathrm{mm}^{3}$ ) (1).

En la fase siguiente, se emplean medios químicos de control y remoción de la placa bacteriana: Solución diluida de clorhexidina al $0,2 \%$ y bicarbonato sódico se prescriben normalmente a los pacientes (1) aplicandose con gasas esterilizadas de algodón.

Por supuesto, para reducir el riesgo de infecciones y hemorragias todas las intervenciones odontológicas son precedidas por profilaxis antibiótica (en general se aconseja un suministro combinado de clindamicina, una cefalosporina y un aminoglucósido), y eventualmente una infusión de trombocitos (hasta alcanzar 40/50.000 trombocitos $\left.\mathrm{mm}^{3}\right)(12,14,21,22)$.

También se puede sugerir una profilaxis antifúngica, mientras la profilaxis antiviral puede utilizarse para los pacientes que producen anticuerpos ANTI-HSV y que tienen que someterse a radiumterapia $(3,12,14$, $21,22,23)$

Para los pacientes que toman fármacos antiblásticos es mejor utilizar el Aciclovir, solo en caso de infección intercurrente $(3,5,14,21,22,23)$.

Por lo tanto durante la terapia antiblástica (Tabla 2) debe suspenderse el cepillado de los arcos dentales y substituirlo con enjuagues con clorhexidina al 0,2\% 3 veces al día o, en algunos raros casos, con una preparación farmacéutica de clorhexidina al 0,2\% 120 ml., nistatina $2,4 \times 10,6$ UI y solución fisiológica $300 \mathrm{ml}$. $(1,2)$

\section{PAPEL DEL ODONTÓLOGO DURANTE EL TRASPLANTE DE MÉDULA ÓSEA (TMO) EN LA REDUCCIÓN DE LA INCIDENCIA DE COIMPLICACIONES PERIODONTALES}

Durante el periodo de la terapia antileucémica, el odontólogo debe actuar para el mantenimiento y la consolidación de todas las medidas preventivas precedentemente adoptadas, ayudando al paciente e instruyendo a los familiares para que colaboren activamente en la motivación diaria de la higiene oral. Se impartirán indicaciones sencillas, fáciles de poner en practica pero que obtienen buenos resultados (Tabla 3).

Cuando es sometido el paciente a trasplante de médula ósea (TMO), las manifestaciones orales son consecuencia de la quimioterapia y de la radioterapia totales al fin de obtener la distrucción de la médula enferma; pudiendo aparecer infecciones, ulceraciones mucosas y xerostomia. El riesgo de infecciones sistémicas o septicemias es elevado y parece tener un origen oral en más del $50 \%$ de los casos documentados $(24,25)$.

Durante la fase inmediatamente sucesiva al trasplante, el paciente puede presentar lesiones causadas por la grave pancitopenia.

Posteriormente, las manifestaciones orales están influenciadas por el uso de la ciclosporina y en este

\section{TABLA 3.- IMEDIDAS PREVENTIVAS PARA EL TIMO}

- Necesaria suspensión de la limpieza mecánica (>inmunodepresión).

- Clorhexidina $(0,12 \%)$ - tres enjuagues al día.

- Enjuagues con bicarbonato sódico varias veces al día. 


\section{TABLA 4.- ALTERACIONES ORALES CLÍNICASS E HISTOLÓGICASS DE LĀ GVHD}

\section{Características}

\section{Características clínicas}

Xerostomia

Reducido flujo salival total

Tumefacción y/o dolor de la parótida

Anomalías sialográficas de la parótida

Sequedad y/o eritema de la mucosa oral

Lesiones liquenoides de la mucosa oral

\section{Características histológicas}

Infiltración linfocitaria difundida de las Gsl

Infiltración linfocitaria periductal de las Gsl

Atrofia y/o distrucción de los ácinos de las Gsl

Infiltración linfocitaria subepitelial en la MB

Degeneración de las células basales de la MB

Sensibilidad (\%)

Especificidad (\%)

61,1

44,4

0,0

27,3

0,0

61,1

88,9

27,8

55,6

64,7

64,7
47,4

63,2

100,0

100,0

100,0

52,6

73,7

100,0

73,7

84,2

94,7 caso el principal efecto secundario es la hipertrofia gingival así como la Graft Versus Host Disease (GVHD), para la que se prescribe precisamente la ciclosporina $(12,15,16,19,20,26)$.

La hiperplasia gingival, aparece en los respondientes dentro de los primeros tres meses desde el inicio de la cura con ciclosporina y el porcentaje varía entre el 25 y el $81 \%$ en función de la dosificación, duración del tratamiento y situación periodontal inicial así como la existencia y tratamiento de posibles enfermedades sistémicas $(27,28)$.

En estos pacientes la encía, no se presenta sólo fibrosa e hiperplástica, sino también muy inflamada. Frente a una situación de este tipo una intervención quirúgica periodontal resultaría problemática si no contraindicada debido a problemas hemorrágicos (9).

En hipertrofia gingival consiguiente a la toma de ciclosporina de pacientes sometidos a TMO, se aconseja el uso de un colutorio de clorhexidina al $0,12 \%$ capaz de bajar del $95 \%$ en pocos días la cuenta bacteriana y fúngica salival hasta suspendiendo el cepillado de los arcos (18).

Útiles son también las soluciones de bicarbonato sódico capaces de remover las seudomembranas y los tejidos necróticos, lubricar las mucosas, elevar el $\mathrm{PH}$ y limitar el crecimiento de la Cándida albicans (14).

Para la hipertrofia gingival debida a ciclosporina se han observado considerables mejoras con el uso de un macrolido: la azitromicina $(1,2,5,10,11,13,15,17$, $18,21)$.

La Graft Versus Host Disease (GVHD) consiste en una reacción del trasplante hacia el huesped por los linfo- citos de la médula trasplantada, que consideran al paciente como extraño, originando una respuesta inmunitaria $(5,10)$. En este caso las formas clinicas orales a cargo de las mucosas y de las glándulas salivales, que se manifiestan en el $80 \%$ de los casos, son similares a las que se registran en numerosas enfermedades autoinmunes como el LES, la esclerodermia, el síndrome de Sjögren o el liquen plano, pudiendo encontrar eritemas, mucositis, gingivitis, xerostomias y lesiones liquenoides de las mucosas $(10,11,12)$ (Tabla 4).

En caso de GVHD, la única terapia consiste en el suministro de cortisonas por su efecto inmunoregulador (2) (Figs. 6, 7, 8).

\section{PAPEL DEL ODONTÓLOGO DESPUÉS DE LAS TERAPIAS ANTILEUCÉIMICAS ("INTERRUPCIÓN DE LA TERAPIA")}

Una vez terminado el ciclo de terapias el odontólogo tendrá que:

- Mantener y reforzar las medidas preventivas adoptadas.

- Revalorizar al paciente cada vez que vuelve al hospital para el control oncológico.

- Continuar las curas odontológicas suspendidas o requeridas por enfermedades sobreañadidas $(3,21$, 23).

El joven paciente hemato-oncológico, que supera con éxito la leucemia, tendrá que ser sometido, después 


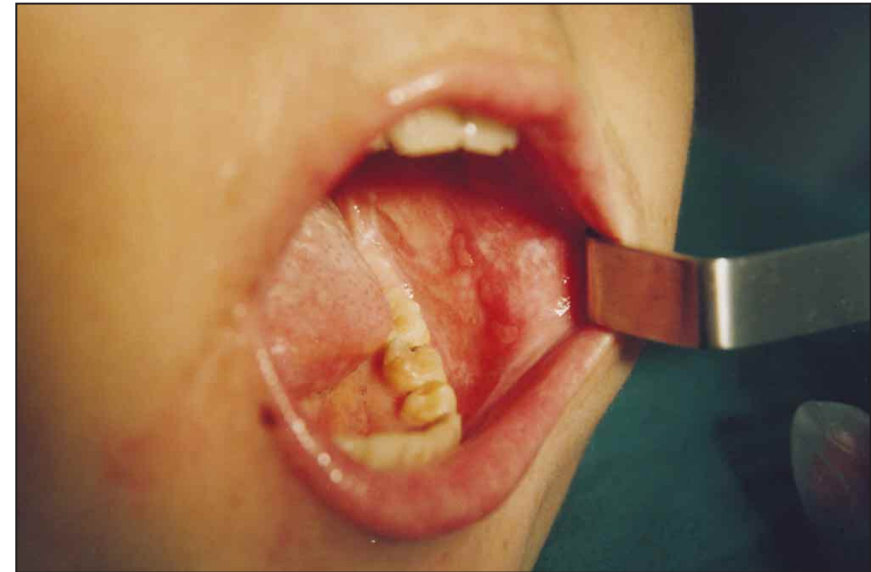

Fig. 6: GVHD de la mucosa oral.

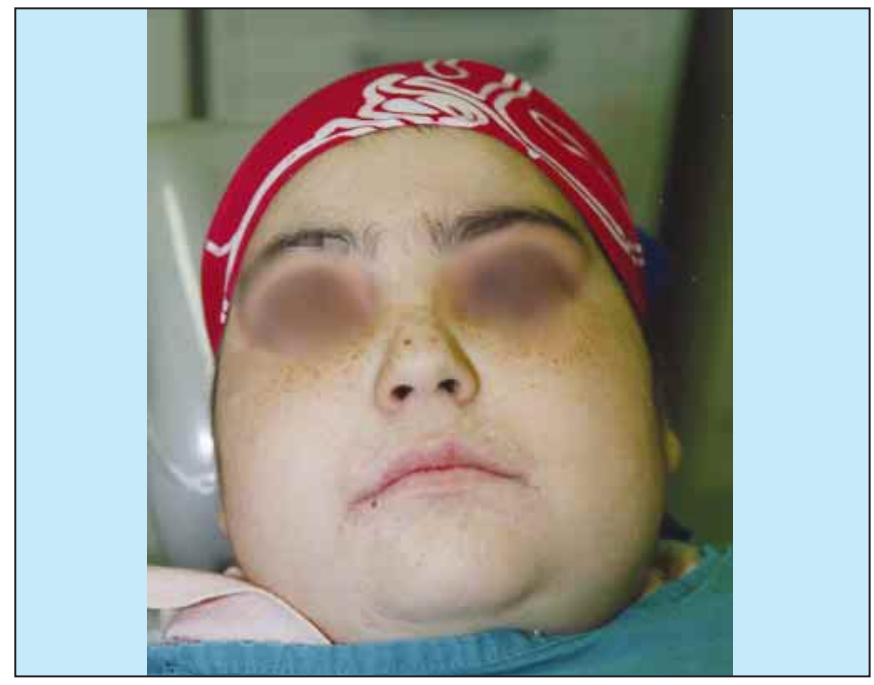

Fig. 7: Paciente sometido a TMO.

\section{TABLA 5.- RESULTADOS DE COMPLICACIONES INFECTIVAS}

\section{CENTRO}

MODOS

LESIÓN

Mucosas

Mucositis

Necrosis de los tejidos blandos (frecuente escisión quirúgica)

Periodontio

\section{Gingivitis}

Periodontitis

Lesiones necrósicas del hueso

\section{Elementos dentales}

Caries/Pulpitis

Rápida destrucción de los tejidos duros del diente y alteraciones del sistema sanguíneo a la pulpa

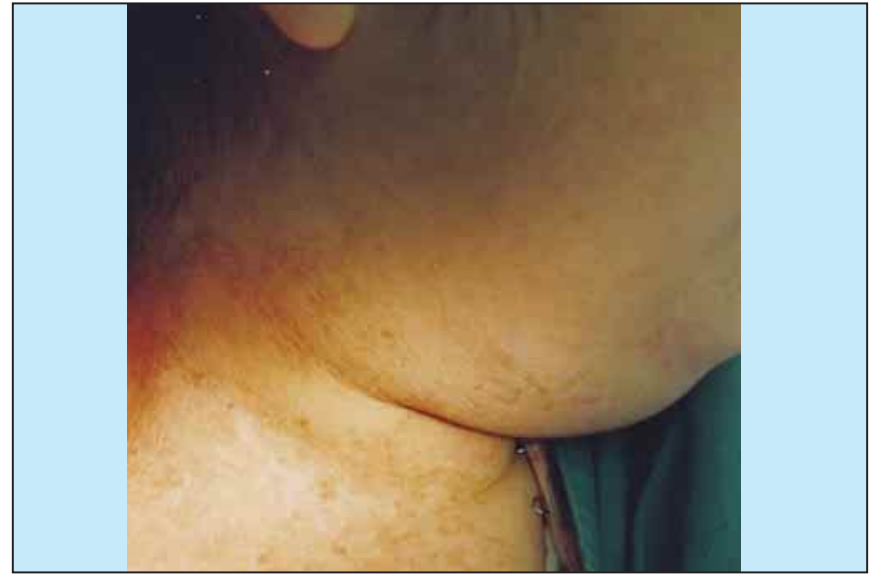

Fig. 8: GVHD cutánea.

\section{TABLA 6.- CITOTOXICIDAD DIRECTA EN CÉLULAS SANAS Y PARCIAL REARREGLO DEL GENOIMA}

Efecto canceroso y mutágeno

Déficit de ACTH

Déficit de GH

Déficit de las hormonas tiroideas

Déficit de cortisol

Alteraciones permanentes de las glándulas salivales

Periodontitis

Alteraciones en la dentición (véase Tabla 8)

Retraso mental

de un tiempo, a evaluaciones odontoestomatológicas de control, al fin de monitorizar posibles alteraciones debidas a la enfermedad.

Existen además manifestaciones a distancia de la cavidad oral, consecuencia de las curas quimioterápicas y radiantes y que interfieren a menudo con el normal desarrollo de los órganos y de las estructuras esqueléticas.

Podemos subdividirlas esquemáticamente en:

- Resultados de complicaciones infectivas (Tabla 5)

- Efecto directo lesivo de los antiblásticos y de la radioterapia (Tabla 6) (14)

\section{EFECTO DIRECTO LESIVO DE LOS ANTIBLÁSTICOS Y DE LA RADIOTERAPIA}

TIPO DE TERAPIA 


\section{MODOS}

LESIÓN

\section{Antiblásticos}

Citotoxicidad en células sanas y parcial rearreglo del genoma. Daño indirecto con alteraciones hipotálamopituitarias (disminución de $\mathrm{GH}$ ).

- Efecto canceroso y mutágeno (de 0,6 a 6,6\%)

- Aparición de patologías autoinmunes (colagenosis)

- Alteraciones de la dentición (véase Tabla 7)

- Alteraciones del crescimiento esquelético (retraso sobre todo en la estatura)

\section{Radioterapia}

Los antiblásticos causan un daño prevalentemente cualitativo en los tejidos duros del diente (sobre todo en dentina y esmalte); la radioterapia provoca daños en el esmalte y sobre todo interfiere con el correcto desarrollo radicular (daños cualitativos y cuantitativos).

Por lo que atañe a la región maxilofacial tenemos un déficit del desarrollo mandibular presente en el $90 \%$ de los pacientes, mientras es importante tener en cuenta que no se registran alteraciones en el desarro- llo de la articulación temporo-mandibular (1).

Precedentes investigaciones de nuestra Sección nos han permetido redactar las Tablas 7 y 8 , de acuerdo con lo descrito por la literatura internacional.

\section{EXPERIENCIA CLÍNICA DE LA SECCIÓN}

Comprobamos la importancia de la estrecha colaboración entre el Centro de Hemato-oncología Hospitalario y una Sección Odontológica selectivamente preparada para tratar a pacientes específicos con necesidades especiales.

Estos pacientes, además de presentar clinicamente problemas hemato-oncológicos, pertenecen a un grupo pediátrico con el inevitable énfasis de las complicaciones operativas.

Un equipo motivado, preparado, disponible y organizado para esos objetivos terapéuticas es la única garantía para el éxito operativo.

Nuestra Sección del Hospital Universitario estudia caso por caso los pacientes señalados por el Centro de Hemato-Oncologia estableciendo un organigrama

\section{TABLA 7.- ALTERACIONES DE LA DENTICIÓN DEBIDASS AL USO DE ANTIBLÁSTICOS}

Alteración

Incidencia (\%)

Elementos

más afectados

Relación con el

periodo de

terapia

Notas

Microdoncía

84

Premolares

Segundos molares

Incisivos laterales

Terceros molares

Coincidencia con

la formación

de la corona

La terapia antes de los 3 años de

edad predispone a la alteración

de la

lámina dental

Amelogenesis

imperfecta (foto 11,12 )

50

\author{
Opacidad del esmalte \\ Hipoplasia \\ Alteración de las \\ líneas incrementales \\ de la dentina
}

Lesión dosis-dependiente

Taurodoncía

5,9

Molares

Premolares

Adelgazamiento radicular
Terapia después de los 9 años $=$ lesiones a las

raíces de los permanentes

Biselado radicular

9-12

Incidencia más alta en niños en terapia de edad $<5$ años

\section{Acortamiento radicular} 30

Distribución según el crecimiento radicular y el momento de la terapia

Retraso eruptivo (foto 13,14 )

Alteraciones de la oclusión 26 


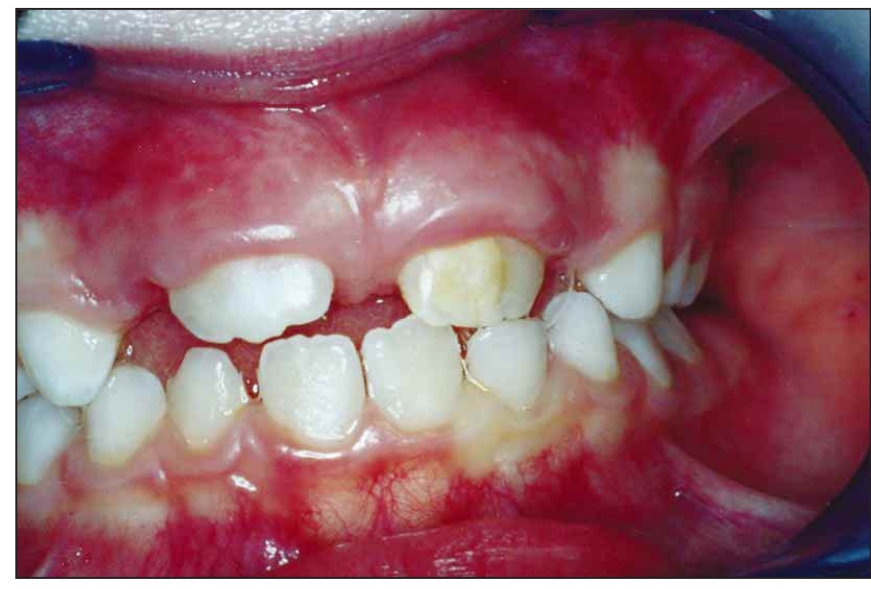

Fig. 9: Amelogenesis imperfecta en el elemento 2.1

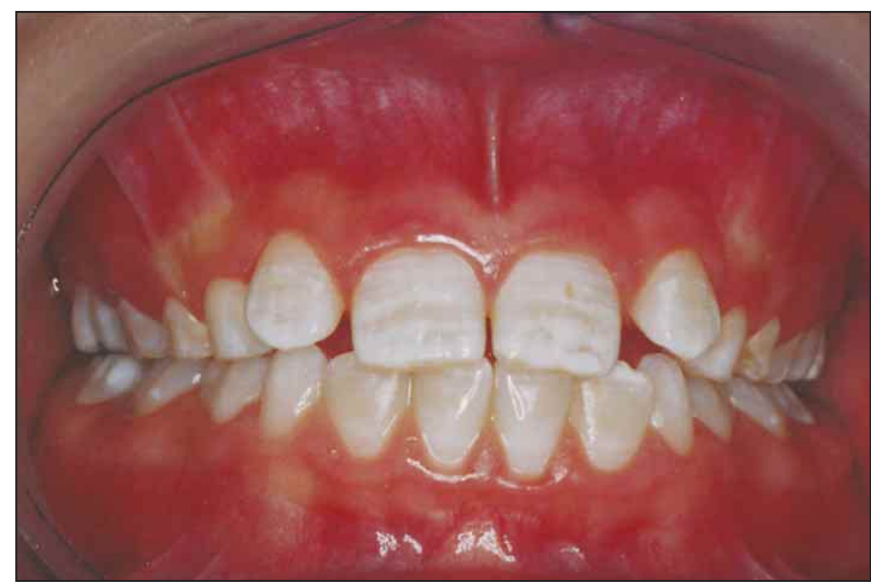

Fig. 10: Amelogenesis imperfecta generalizada

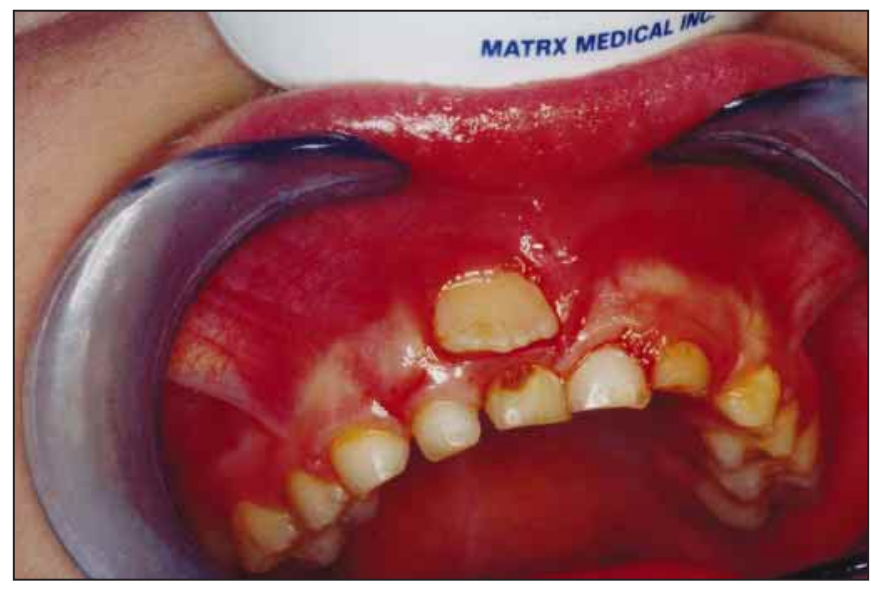

Fig. 11: Retraso eruptivo debido al uso de antiblásticos y/o radioterapia

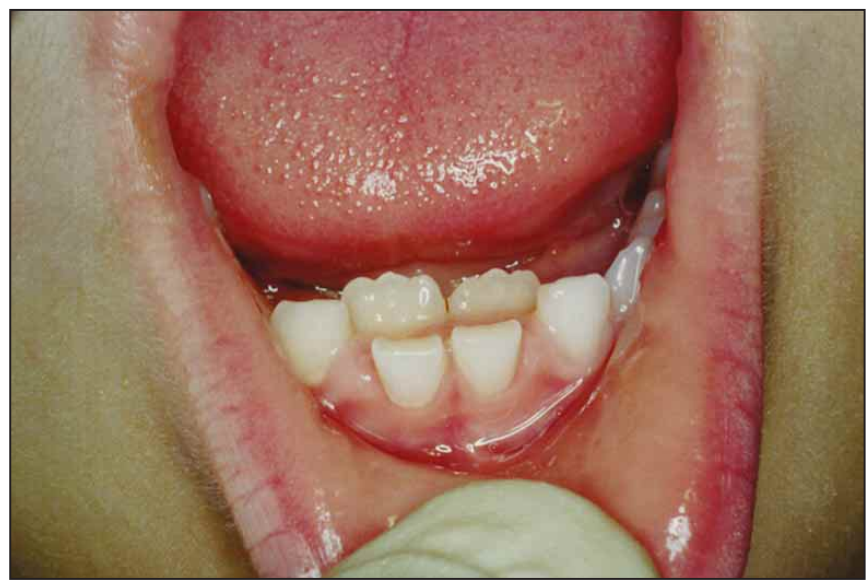

Fig. 12: Retraso eruptivo debido al uso de antiblásticos y/o radioterapia

\section{TABLA 8.- ALTERACIONES DE LA DENTICIÓN A CONSECUDNCIA DE RADIOTERAPIA}

\begin{tabular}{|c|c|c|c|c|}
\hline Alteración & Incidencia (\%) & Elementos más afectados & Relación con el período de terapia & Notas \\
\hline Microdoncia & 20 & $\begin{array}{l}\text { Premolares. Segundos molares. } \\
\text { Incisivos laterales. Terceros molares }\end{array}$ & $\begin{array}{l}\text { Más frecuente en niños de } \\
\text { edad }<5 \text { años }\end{array}$ & $\begin{array}{l}\text { Más frecuente para } \\
\text { dosis de } 2400 \mathrm{cGy}\end{array}$ \\
\hline Agenesias & $8,5-2,5$ & $\begin{array}{l}\text { Incisivos laterales. Premolares } \\
\text { Segundos molares }\end{array}$ & $\begin{array}{l}\text { Más frecuente en niños de } \\
\text { edad }<5 \text { años }\end{array}$ & $\begin{array}{l}\text { Más frecuentes para } \\
\text { dosis de } 2400 \mathrm{cGy}\end{array}$ \\
\hline $\begin{array}{l}\text { Hipoplasia del } \\
\text { esmalte }\end{array}$ & 95 & Elementos del maxilar superior & $\begin{array}{l}\text { Más frecuente en niños de } \\
\text { edad < } 5 \text { años }\end{array}$ & $\begin{array}{c}\text { Más frecuente para } \\
\text { dosis de } 1800-2400 \\
\text { cGy }\end{array}$ \\
\hline $\begin{array}{l}\text { Alteración del } \\
\text { número de raíces }\end{array}$ & $54-57$ & & & $\begin{array}{c}\text { Más frecuentes para } \\
\text { dosis superiores a } \\
1800 \mathrm{cGy}\end{array}$ \\
\hline \begin{tabular}{|l|} 
Taurodoncia \\
\end{tabular} & 5,7 & Molares. Premolares & & \\
\hline Acortamiento radicular & $64-100$ & & & $\begin{array}{l}\text { Dosis y edad } \\
\text { dependientes }\end{array}$ \\
\hline Biselado radicular & $36-100$ & & & $\begin{array}{l}\text { Dosis y edad } \\
\text { dependientes }\end{array}$ \\
\hline \begin{tabular}{|l|} 
Retraso eruptivo \\
(foto 13-14)
\end{tabular} & 4 & & & \\
\hline
\end{tabular}


preciso de tiempos y métodos de intervención diferentes, según los casos.

Esos datos son analizados y elaborados para obtener una optimización ideal del tiempo disponible, haciendo que los tiempos operativos odontológicos coincidan con los tiempos terapéuticos de la leucemia.

En cada fase hay que tener en cuenta la disponibilidad psicológica de los jóvenes pacientes, ya por sí muy comprometida, y la disponibilidad de la familia, que a menudo procede de lugares pobres y lejanos.

La primera visita del paciente se efectúa directamente en DH (centro de día hemato-oncológico) con las siguientes ventajas:

- Posibilidad de visitar al paciente cuando el diagnóstico de leucemia es muy reciente.

- Posibilidad de hacer una evaluación odontológica antes del tratamiento antineoplásico.

- Posibilidad de hacer evaluaciones odontológicas precisas sin tener que hacer frente a emergencias odontológicas en pacientes de riesgo como el paciente leucémico.

- Posibilidad de consolidar una relación de colaboración entre la Sección Odontológica y División Hospitalaria.

Durante la primera visita, en centros de día, en presencia del personal médico de la división es posible discutir juntos de la historia clínica del paciente, de la leucemia y de las exigencias odontológicas. La respuesta del joven paciente y de su familia en general es siempre positiva: en este momento los padres se encuentran en una fase particularmente receptiva por lo que atañe a la salud de su hijo, incluyendo la información y la motivación a las curas odontológicas.

Por eso, después de haber recogido datos anamnésicos, evaluado las condiciones sistémicas del paciente y unificado un plan de tratamiento odontológico, se le introduce al joven paciente en el organigrama de la Sección Odontológica para que se someta a las curas.

\section{MATERIALES Y IMÉTODOS}

Este tipo de organización nos ha permitido en, aproximadamente, dieciocho meses de trabajo llevar a cabo un gran número de intervenciones terapéuticas siguiendo esquemas pedodónticos y controlando sobre todo las enfermedades periodontales.
Se trata de una experiencia única a nivel regional y poco representada a nivel nacional, que ha permetido a la Sección de Hemato-oncologia Odontológica del Hospital San Gerardo-Universidad de Milán-Bicocca convertirse en poco tiempo en un punto de referencia para muchos pacientes, incluso procedientes de regiones lejanas.

La atención de la Sección se ha dirigido también al empleo, investigación y desarrollo de nuevos materiales odontológicos que mejoren las condiciones operativas y de mantenimiento a medio plazo de los jóvenes pacientes.

Los tipos de terapias propuestas y llevadas a cabo en la Sección de Odontología Hemato-oncológica comprenden:

- Remoción del cálculo

- Profilaxis con flúor

- Selladores

- Tratamiento farmacológico con clorhexidina (0,12\% o $0,2 \%$ )

- Empastes

- Pulpotomías y desvitalizaciones

- Avulsiones

- Alisados

- Frenulectomías

- Chequeo ortodóntico.

\section{RESULTADOS}

Los jóvenes pacientes en terapia en la Sección de Hemato-Oncología tenían una edad comprendida entre los 4 y los 13 años, cuyas enfermedades se habían diagnosticado entre 1992 y 2001 (Gráfico 2).

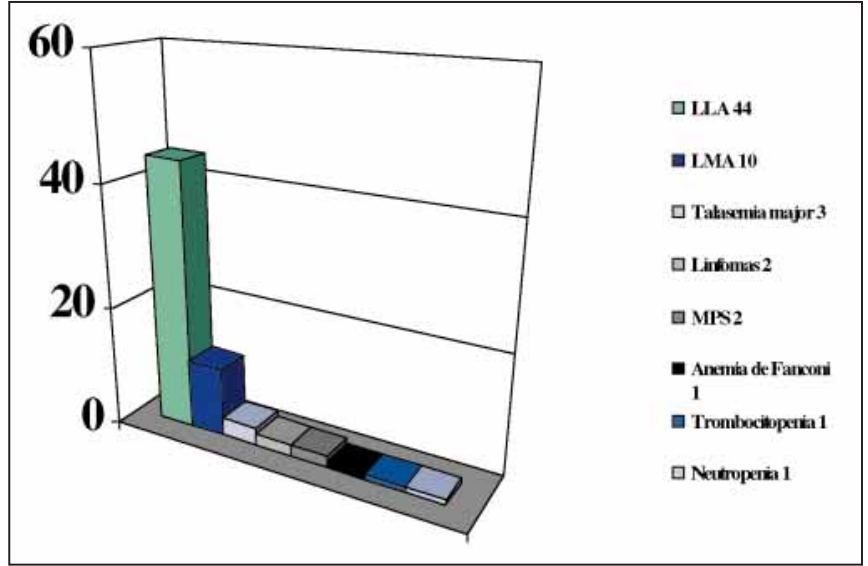

Gráfico 2: Número de pacientes sometidos a terapia en función de las patologías hemato-oncológicas diagnosticadas. 


\section{INCIDENCIA DEL LAS ENFERIMEDADES HEMATO-ONCOLÓGICAS}

Particular atención siempre se ha dirigido a la prevención de las infecciones locales y de las septicemias tanto en el periodo pre como post-terapia antileucémica.

La medidas preventivas llevadas a la práctica comprendían, según el tratamiento odontológico y las condiciones generales del paciente, prescripción de enjuagues con clorhexidina y bicarbonato sódico, profilaxis antibiótica (clindamicina, cefalosporina y aminoglucósido), profilaxis antiviral (Aciclovir), profilaxis antifúngica (fluconazol).

En particular esas medidas preventivas se realizaban cuando el paciente tenía que ser sometido a remoción del cálculo, tartrectomía, terapias endodónticas, terapias conservativas con utilización de matrices y/o hilos retractores (pues con posible sangrado) y por supuesto en caso de avulsiones, frenulectomías y terapias quirúgicas periodontales.

En todos los demás casos no hemos realizado ningún tipo de prevención.

En los pacientes en los que resultaba peligroso todo tipo de operaciones, debido al momento de la terapia antileucémica, se recurría a un "tratamiento farmacológico" mediante enjuagues con clorhexidina $(0,2 \%$ o $0,12 \%$ ) como coadyuvante en el control de la carga bacteriana.

Particular atención se ha dirigido al control del estado de higiene oral y de las condiciones periodontales del joven paciente.

Se diferencian eventuales estados de inflamación gingival fisiológica, como los debidos a la erupción dental o al cambio, de otros estados de sufrimiento periodontal de carácter diferente (por ejemplo infiltración leucémica gingival, periodontitis por placa o por inmunodepresión).

Las condiciones de higiene oral se pueden estudiar mediante índices de placa, cálculo y sangrado, que miden indirectamente también el grado de gingivitis.

No se lleva a cabo sondeo periodontal (se considera que un sondeo periodontal subgingival sería extremamente inoportuno en pacientes en los que ya el estado de salud general es muy débil, ya que podría causar fenómenos hemorrágicos e infectivos en sujetos inmunocomprometidos; por eso se prefieren índices normalizados de placa y de cálculo menos invasivos, como por ejemplo OHI-S, VPI de Bay y Aynamo, index OMS).
Hay que recordar que en el paciente leucémico la hipertrofia gingival por infiltración leucémica o una gingivitis hipertrófica reactiva pueden llevar a la formación de seudo-sacos, receptáculo de residuos alimentares y bacterias.

El estudio de la morbilidad de cada diente se lleva a cabo poniendo el mango del espejo en la superficie vestibular del elemento dental y el dedo en la superficie oral. Una escala de 1 a 3 (index de Miller) distingue una movilidad apenas perceptible, una movilidad inferior a $1 \mathrm{~mm}$ en sentido vestíbulo lingual y una movilidad superior a $1 \mathrm{~mm}$, con la posible presencia de movilidad también vertical. En el paciente leucémico una movilidad dental incluso grave puede ser causada por:

- Reabsorción ósea de carácter inflamatorio (a consecuencia de periodontitis necrotizante o de periodontitis con inicio precoz).

- Infiltración leucémica a nivel del periodonto y/o del hueso alveolar.

- Infecciones odontógenas periapicales (absceso).

- Osteomielitis.

En el paciente en "interrupción de terapia" la hipermovilidad puede depender de factores fisiológicos (raiz en formación) o de factores patológicos (alteraciones radiculares y/o óseas verosímilmente dependientes del suministro de antiblásticos y radioterapia).

De las 164 obturaciones realizadas, aproximadamente la mitad ha requerido una profilaxis de las infecciones ya que necesitava el uso de una matriz y/o del hilo retractor.

Poniendo en práctica estas reglas en nuestra experiencia, a pesar de la casuística descrita por la literatura, no hemos registrado casos de septicemías.

Por fin hemos introducido intencionadamente el chequeo ortodóntico entre los tratamientos propuestos y realizados, pues creemos que esto tenga un considerable papel interceptivo en las manifestaciones de las alteraciones dento-esqueléticas, en particular en estos jóvenes pacientes (Gráfico 3).

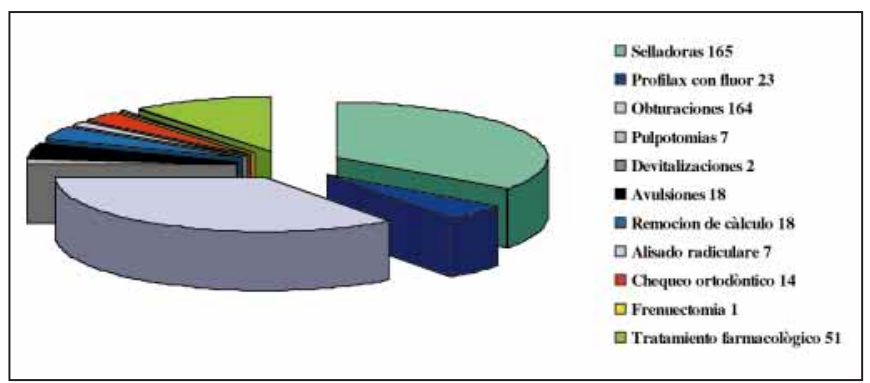

Gráfico 3: Terapias odontológicas realizadas en 18 meses. 


\section{AVANCES}

Volumen 17 - No 2 - Agosto 2005

\section{OPERATIVIDAD CLÍNICA}

Analizando estos datos se desprende que el mayor papel terapéutico desempeñado por la Sección es de tipo preventivo de las complicaciones sistémicas de la enfermedad y de las terapias antiblásticas y radiantes. En particular la Sección se ha ocupado de dos aspectos fundamentales:

- Periodontología: prevenir hipertrofias gingivales, infecciones mucosas y gingivales, etc.

- Ortodoncia: individualizar alteraciones y enfermedades esqueléticas y además monitorizar la situación dental.

Sobre todo por lo que atañe al primer aspecto, nuestra Sección se ha valido de protocolos modificados de higiene y profilaxis, en tema de prevención de las enfermedades periodontales en pacientes de edad 3-15 años, utilizados por el Centro de Higiene Oral (CIO) de la Clínica Odontológica de la Universidad de Milan-Bicocca del Hospital San Gerardo de Monza, resumidos en la Tabla 9 (Fotos $13,14,15)$.

\section{DISCUSIÓN}

El estudio de pacientes leucémicos que presentan problemas odontológicos y periodontales ha sido el principal objetivo de este trabajo de investigación.

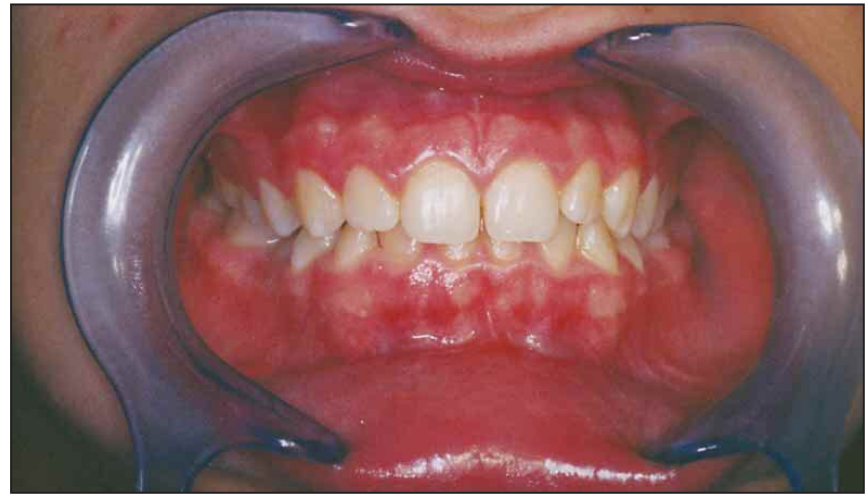

Fig. 13: Paciente pediátrico leucémico con presencia de placa supra-gingival

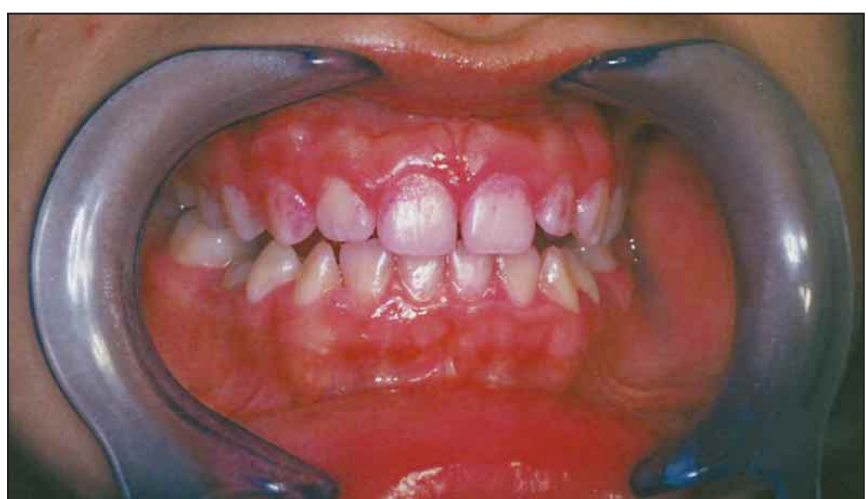

Fig. 14: La placa se pone en evidencia mediante pastilla detectora

De hecho sa ha visto que la leucemia, junto a la terapia antiblástica, crea una situación clínico-odontológica a

\section{TABLA 9.- PROTOCOLOS DE HIGIENEY PROFILAXIS}

Cita 1 de 60': Código 1

Higiene oral: remoción del cálculo supra-gingival y de la placa

Lección de métodos de higiene oral

Cita 2 de 40': Código 2

Evaluación del index de placa, limpieza individual

Motivación y lección de higiene oral

Cita 3 de 40': Código 3

Lustre y aplicación de flúor

Fijar cita de control después de 2 meses

Cita de control de 45':

Código R (con número en secuencia negro o rojo según la situación clínica favorable o desfavorable)

Visita

Evaluación index de placa (IP)

Si el IP es bueno, se pasa a un lustre y a una aplicación de flúor y por eso se fijan citas de control cada 4 meses

Si el IP no es bueno, se hace una remoción reforzando las motivaciones a la higiene oral y se fijan dos citas a distancia de una semana análogas a los códigos 2 y 3 


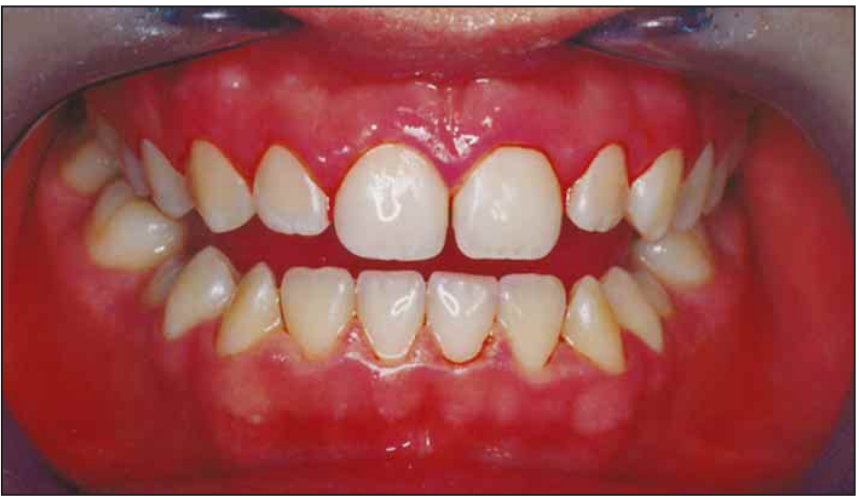

Fig. 15: El joven paciente después de la preparación inicial

menudo degenerativa, sobre todo en el joven paciente, donde los procesos hormonales de desarrollo, alterados o suspendidos por la terapia, pueden crear problemas morfo-formativos de los arcos dentales.

También las alteraciones de los tejidos blandos del cavo oral pueden producir condiciones predisponentes para la llegada de enfermedades con complicaciones a menudo graves, como las septicemias.

Por lo tanto la creación de un núcleo odontológico especializado puede ser por supuesto una importante ayuda en todas las fases de la leucemia.

\section{CONCLUSIONES}

El tratamiento odontológico de los pacientes pediátricos afectados por leucemia tiene que ser llevado a la práctica por sanitarios preparados. La estrecha colaboración entre la Pediatría Hemato-oncológica y la Clínica Odontológica es una premisa indispensable para la curación de los jóvenes pacientes. Sólo una atenta observación podrá permitir un diagnóstico clínico de las alteraciones dento-periodontales que ocurren en el cavo oral a consecuencia de la leucemia y de las terapias puestas en acto:

- Manifestaciones orales al inicio de la leucemia hipertrofia gingival, NUMB CHIN SYNDROME, infecciones, hemorragias gingivales, etc;

- Manifestaciones orales a consecuencia de las terapias anti-neoplásicas y sobre todo del TMO - mucositis, periodontitis, caries graves, xerostomia, infecciones bacterianas, virales y fungicas, hemorragias, displasias mucosas, lesiones orales por GVHD, hiperplasia gingival por ciclosporina;

- Manifestaciones orales a largo plazo en pacientes sobrevivientes: alteraciones tanto de carácter cualitativo como cuantitativo de la fórmula dental, hipoplasia mandibular.
En estos pacientes será extremamente importante enseñar y sensibilizar (junto a la familia) sobre las problemáticas odontológicas. La familia, de hecho, como está obsesionada en el problema oncológico, puede propender a pasar por lo alto los problemas odontológicos del niño, y esto puede aumentar el riesgo de infecciones orales y de difusión sistémica de micro-organismos patógenos. Tal riesgo se puede reducir considerablemente con simples, rápidos e indoloros métodos preventivos realizados y decididos también en colaboración con el CIO.

La creación de un equipo que trabaje en estrecha colaboración y respaldado por la División Hospitalaria de Hemato-oncología Pediátrica responde pues a la exigencia de integrar los conocimientos médicos especializados en el ámbito hemato-oncológico con los odontológicos. Esto para garantizar a estos jóvenes pacientes la salud del área oral, fundamental por sus funciones fisiológicas y ademas por su papel psicológico y social.

\section{SUIMIMARY}

The aim of this work is to assess tooth-periodontal complications of pediatric patients affected by leukemia.

Importance will be addressed to the dentist role in the first steps of the disease with particular attention to the diagnosis of periodontal and neurologic associated syndromes.

Authors talk about periodontal complication and dental-skeletal alterations linked to antiblastic therapy, too. They expose how and what is done for these patients in the hemato-oncology dentistry public structure where they play an active role.

Finally, they expose the pathologic percentiles recorded (18 months period).

\section{KEY WORDS}

Leukemia, tooth-periodontal oral manifestation of leukemia, department of hemato-oncology dentistry, prevention and oral hygiene

\section{CORRESPONDENCIA}

Universidad de Milán - Bicocca

Facultad de Medicina y Cirugía Clínica Odontológica 


\section{BIBLIOGRAFIA}

1. M. Baldoni, L. Balucchi, C. Bialetti, A. Mingardi. Gestione dei pazienti pediatrici affetti da leucemia. Il Dentista Moderno 2000; 5: 143-59.

2. O'Sullivan EA, Duggals MS et al. Changes in the oral microflora during cytotoxit chemotherapy in children being treated for acute leukemia. Oral Surg Oral Med Oral Pathol 1993; 76 (2): 161-8.

3. De Beule F, Bercy P et al. The effectiveness of a preventive regimen on the periodontal health on patients undergoing chemotherapy for leukemia and lymphoma. J Clinic Periodontol 1991; 18: 346-7.

4. Balucchi L, Rebagliati E, Baldoni M. Numb chin sindrome: linee guida per l'odontoiatra. Il Dentista Moderno 2001; 4: 113-26.

5. Epstein JB, Ransier A et al. Prophilaxis of candidiadis in patients with leukemia and bone marrow transplants. Oral Surg Oral Med Oral Pathol Oral Radiol Endod 1996; 81: 291-6.

6. Casamassimo PS, Bronstein SL, Christensen J, Fields H. Periodontal diseases and temporomandibular joint disorder. In: Pinkham JR (Hrsg). Pediatric dentistry. Infancy through adolescence. Philadelphia: Sauders; 1985.

7. Magnusson BO, Matsson L, Modeèr T. Gingivitis and periodontal disease in children. In: Magnusson BO (Hrsr). Pedodontics. A systematic approach. Kopenhagen: Munksgaard; 1981.

8. Kristoffersen T, Trygge L. Necrotizing gingivitis. In: Lindhe J (Hrsg). Textbook of clinical periodontology. Kopenhagen: Munksgaard; 1989.

9. Scully C, Cawson RA. Il trattamento odontoiatrico nei pazienti affetti da malattie sistemiche. Roma: Antonio Delfino Editore; 2000.

10. Nakamura S et al. Oral involvement in chronic graft host disease after allogenic bone marrow transplantation. Oral Surg Oral Med Oral Pathol Oral Radio Endod 1996; 82: 556-63.

11. Hovi L, Saarinen UM. Opportunistic osteomyelitis in the jaws of children in immunosuppressive chemotherapy. J Pediatric Hematol Oncol 1996; 18 (1): 90-4.

12. Peterson DE. Pre-treatment strategies for infection prevention in chemotherapy patients. NCI Monography 1990; 9: 61-71.

13. Bunetel L, Bonnaure-Mallet M. Oral pathoses caused by candida during chemotherapy. Oral Surg Oral Med Oral Pathol Oral Radiol Endod 1996; 82: 161-5.

14. Committee on Research Science and Therapy of the American Academy of Periodontology. Periodontal con siderations in the management of the cancer patient. J Periodontol 1997; 68 (8): 791-801.

15. Puig JM, Lloveras J et al. Treatment of gingival hyperplasia secondary to cyclosporine by the new madrolide azitromycin. Transplant Proc 1997;29(5):2379-80.

16. Russel RG, Graveley R et al. The effect of cyclosporin A on bone and cartilage. $\mathrm{Br} \mathrm{J}$ Reumatol 1993; 3 (1): 42-6.

17. Barret AP, Shifter M. Antibiotic strategy in oro-facial, head and neck infections in severe neutropenia. Oral Surg Oral Med Oral Pathol 1994; 77 (4): 350-5.

18. Bondi E, Baroni C et al. Trapianto di midollo nei bambini: trattamento delle complicanze orali. Dent Cadmos 1993; 16: 72-7.

19. Borel JF, Feurier C et al. Effect of the new antylymphocyte peptide cyclosporine $A$ in animals. Immunology 1997; 32: 1017-22.

20. Pajari U, Ollila $P$ et al. Incidence of dental caries in children with acute lymphoblastic leukemia is related to the therapy used. J Dent Child 1995; 62 (5): 349-52.

21. Wright WE, Haller JM et al. An oral disease prevention program for patients receiving radiation and chemotherapy. JADA 1985; 110: 43-7.

22. Balucchi L, Baldoni M. Protocollo operativo e di ricerca. Dental abnormalities in children treated for acute lymphoblastic leukemia. Corso di Parodontologia, CLOPD, Università di Milano.

23. Epsein JB, Van der Mejj E et al. Effect of compliance with fluoride gel application on caries and caries risk in patient after radiation therapy for head and neck cancer. Oral Surg Oral Med Oral Pathol Oral Radiol Endod 1992; 82: $268-75$

24. Childers NK, Stinnet EA, Wheeler $P$, Wright JT, Castelberry RP, Dasanayake AP. Oral complications in children with cancer. Oral Surg Oral Med Oral Pathol 1993 Jan; 75 (1): $41-7$.

25. Cousin GC. Oral manifestation of leukemia. Dent Update 1997 Mar; 24 (2): 67-70.

26. Katsung BG. Basic and clinical pharmacology. Cancer chemotherapy. Chap. 56. Salomon SE, Sartorelli AC (eds), Appleton \& Lange; 1989: 683-703.

27. Seymour RA, Smith DG, Roger SR. The comparative effect of azathiopine and cyclosporin on some gingival health parameters of renal transplant patients. J Clin Periodont $1987 ; 14: 610-3$.

28. Seymour RA, Jacobs DJ. Cyclosporin and the gingival tissues. J Clin Periodont 1982; 19: 1-11. 